\title{
DE ESTOCOLMO (1972) A RÍO + 20. \\ “EL FUTURO QUE QUEREMOS”
}

\section{Carlos Aníbal Rodríguez ${ }^{1}$}

I. Hace cuarenta (40) años se iniciaba el Derecho Ambiental en la Conferencia de Estocolmo (1972).

1. El hombre es a la vez obra y artífice del medio que lo rodea, el cual le da el sustento material y le brinda la oportunidad de desarrollarse intelectual, moral, social y espiritualmente. En la larga y tortuosa evolución de la raza humana en este planeta se ha llegado a una etapa en que, gracias a la rápida aceleración de la ciencia y la tecnología, el hombre ha adquirido el poder de transformar, de innumerables maneras y en una escala sin precedentes, cuanto lo rodea. Los dos aspectos del medio humano, el natural y el artificial, son esenciales para el bienestar del hombre y para el goce de los derechos humanos fundamentales, incluso el derecho a la vida misma.

Luego vino la Declaración de Río de Janeiro de 1992 y los demás documentos de esa magna conferencia.

En 2002 se celebró la cumbre mundial sobre el Desarrollo Sustentable en Johannesburgo

Y finalmente la esperada conferencia de Río+ 20 celebrada en junio de 2012.

Se concreta así el cuerpo que da fundamento al Derecho Ambiental, el que más se ha desarrollado en los últimos años.

Es un derecho invasivo y reglamentario de Derechos Humanos fundamentales, es decir los Derechos Ambientales.

Anteriores a la Reforma de la Constitución Nacional de 1.994, que el constituyente no hizo -en palabras de la Corte Suprema de Justicia de la Naciónsino enumerarlos y darles el carácter constitucional.

${ }^{1}$ Profesor Titular p/concurso de Seminario de Integración de Derecho Ambiental y de los Recursos Naturales. 
El reconocimiento de status constitucional del derecho al goce de un ambiente sano, así como la expresa y típica previsión atinente a la obligación de recomponer el daño ambiental no configuran una mera expresión de buenos y deseables propósitos para las generaciones del porvenir, supeditados en su eficacia a una potestad discrecional de los poderes públicos, federales o provinciales, sino la precisa y positiva decisión del constituyente de 1994 de enumerar y jerarquizar con rango supremo a un derecho preexistente, que frente a la supremacía establecida en el art. 31 de la Constitución Nacional y las competencias regladas en el art. 116 de esta Ley Fundamental para la jurisdicción federal, sostienen la intervención de este fuero de naturaleza excepcional para los asuntos en que la afectación se extienda más allá de uno de los estados federados y se persiga la tutela que prevé la Carta Magna. (CSJN, "Mendoza, Beatriz s. y otros c. Estado Nacional y otros”, fallo del 20/06/2006, M.2771.XLI)

Tan es así que el proyecto de reforma del Código Civil y Comercial al respecto dice: “Artículo 241. Jurisdicción. Cualquiera sea la jurisdicción en que se ejerzan los derechos, debe respetarse la normativa sobre presupuestos mínimos que resulte aplicable”.

Va suyo que se reconoce expresamente la jerarquía superior de estos derechos humanos ambientales.

II. La Conferencia de las Naciones Unidas sobre el Desarrollo Sostenible se realizó en Río de Janeiro (Brasil) entre los días 20 a 22 de junio de 2012 ha emitido una documento final de 283 puntos sobre el destino del ambiente y de los seres humanos que somos parte de este planeta Tierra.

III. En la conferencia estuvieron los representantes de 191 países, incluidos 57 jefes de Estados.

El contexto económico, político y social internacional en que se desarrolló esta conferencia internacional que fijará lo política ambiental de los próximos años se realizó en un clima de crisis similar a la que sobrevino a la "gran depresión” iniciada en 1929 y que se extendió a nivel global por lo menos hasta el inicio de la 2da. Guerra Mundial en 1939 y para otros hasta 1946. 
Esta crisis iniciada en 2.007 por las hipotecas "subprime” y luego extendida al sistema bancario y a la economía real, que al momento de la Conferencia estaba en eclosión en Europa.

Difícilmente podíamos esperar que los países desarrollados o con mayor poder en el mundo tendrían como prioridad el ambiente cuando están luchando denodadamente con economías que se derrumban, alto desempleo e incertidumbre generalizada sobre el futuro del mundo tal cual lo conocemos hoy.

$I V$. La gran mayoría de los que los que se dedican a las cuestiones ambientales, seguramente dirán que la Declaración final denominada: "el futuro que queremos" no es más que un largo rosario de buenas intenciones, supeditadas a la voluntad de los gobiernos de los Estados o tal vez como decimos los operadores del derecho como "Soft law".

Sin perjuicio de ello quiero rescatar en este trabajo los aspectos que considero positivo de tal declaración, por sobre todas las cosas, para su aplicación en los casos concretos que se presenten en el futuro de acuerdo a nuestra actual normativa constitucional en virtud de la reforma constitucional de 1994.

V. La Declaración ratifica plenamente los distintos documentos referidos a la protección ambiental y a la erradicación de la pobreza como el mayor problema que afronta el mundo en la actualidad y una condición indispensable del desarrollo sostenible (p. 2).

En ellos se incluyen documentos que son consecuencia del calentamiento global, la financiación para el desarrollo, los objetivos de Desarrollo del Milenio de la UN y los documentos referidos al problema del aumento de la población mundial.

Así se dice:

16. Reafirmamos nuestro compromiso de aplicar íntegramente la Declaración de Río sobre el Medio Ambiente y el Desarrollo, el Programa 21, el Plan para la ulterior ejecución del Programa 21, el Plan de Aplicación de las Decisiones de la Cumbre Mundial sobre el Desarrollo Sostenible (Plan de Aplicación de las Decisiones de Johannesburgo) y la Declaración de Johannesburgo sobre el Desarrollo Sostenible de la Cumbre Mundial sobre el Desarrollo Sostenible, el Programa de Acción para el desarrollo sostenible de los pequeños Estados insulares en desarrollo (Programa de Acción de Barbados) y la Estrategia de Mauricio para la ejecución ulterior 
del Programa de Acción para el desarrollo sostenible de los pequeños Estados insulares en desarrollo. Reafirmamos también nuestro compromiso de aplicar íntegramente el Programa de Acción a favor de los países menos adelantados para el decenio 2011-2020 (Programa de Acción de Estambul), el Programa de Acción de Almaty: atención de las necesidades especiales de los países en desarrollo sin litoral dentro de un nuevo marco mundial para la cooperación en materia de transporte de tránsito para los países en desarrollo sin litoral y de tránsito, la Declaración política sobre las necesidades de desarrollo de África, y la Nueva Alianza para el Desarrollo de África. Recordamos asimismo nuestros compromisos respecto de los resultados de todas las grandes conferencias y cumbres de las Naciones Unidas en los ámbitos económico, social y ambiental, incluida la Declaración del Milenio de las Naciones Unidas, el Documento Final de la Cumbre Mundial 2005, el Consenso de Monterrey de la Conferencia Internacional sobre la Financiación para el Desarrollo, la Declaración de Doha sobre la financiación para el desarrollo, el documento final de la Reunión Plenaria de Alto Nivel de la Asamblea General de las Naciones Unidas sobre los Objetivos de Desarrollo del Milenio, el Programa de Acción de la Conferencia Internacional sobre la Población y el Desarrollo, las medidas clave para seguir ejecutando el Programa de Acción de la Conferencia Internacional sobre la Población y el Desarrollo, y la Declaración y Plataforma de Acción de Beijing.

Es decir la plena vigencia de lo que constituye hasta este momento la base del Derecho Internacional Ambiental, relacionado estrechamente con los Derecho Humanos.

Además de la exigencia de aquellos convenios de cumplimiento obligatorio, en especial aplicando el principio de cooperación internacional, a saber:

17. Reconocemos la importancia que revisten los tres Convenios de Río para el fomento del desarrollo sostenible y a este respecto instamos a todas las Partes a que cumplan íntegramente los compromisos contraídos en virtud del Convenio Marco de las Naciones Unidas sobre el Cambio Climático, el Convenio sobre la Diversidad Biológica y la Convención de las Naciones Unidas de Lucha contra 
la Desertificación en los Países Afectados por Sequía Grave o Desertificación, en particular en África, de conformidad con sus respectivos principios y disposiciones, así como a que tomen medidas eficaces y concretas en todos los niveles y a que intensifiquen la cooperación internacional.

VI. Luego de reconocer que los avances en materia del ambiente desde la Conferencia de Río de 1992 fueron desiguales, resalta nuevamente la cuestión del aumento de la población mundial y sus consecuentes problemas:

21. Nos preocupa profundamente el hecho de que una de cada cinco personas de este planeta, es decir, más de 1.000 millones de personas, siga viviendo en la extrema pobreza, y que una de cada siete, o el 14\%, esté malnutrida, en tanto que ciertos problemas de salud pública, como las pandemias y las epidemias, siguen constituyendo una amenaza omnipresente. En este contexto, tomamos nota de los debates en curso sobre la seguridad humana en la Asamblea General. Reconocemos que, dado que para 2050 se proyecta una población mundial superior a 9.000 millones de personas y que se estima que las dos terceras partes de esa población vivirá en las ciudades, debemos intensificar los esfuerzos orientados a lograr el desarrollo sostenible, y en particular, la erradicación de la pobreza, el hambre $y$ las enfermedades evitables.

VII. Los derechos ambientales son Derecho Humanos esenciales o fundamentales:

Como sabemos en nuestro país existe un sistema de doble legalidad, constitucional. Por una parte la dogmática constitucional, esto es la Declaración de Derechos y Garantías de la CN de 1853/1860 y por otro, diez Tratados Supranacionales de DD.HH. incorporados a la CN por vía del artículo 75, inc. 22 y otros 2 jerarquizados posteriormente por decisión del Congreso Nacional con las mayorías exigidas por la norma en cuestión. ${ }^{2}$

Además de ellos los tratados de integración regional (Art.75, inc. 24º de la CN).

${ }^{2}$ La «Convención interamericana sobre desaparición forzada de Personas aprobada por la Asamblea General de la O.E.A” (Ley 24820) y la «Convención sobre imprescriptibilidad de los crímenes de guerra y de los crímenes de lesa humanidad» (Ley 25778). 
En un segundo nivel de esta pirámide trunca que es hoy el Orden Jurídico Argentino, se encuentra la llamada supralegalidad infraconstitucional. En este nivel se ubican los demás Tratados, Pactos y Convenciones suscriptos por el país.

En el tercer nivel del Orden Jurídico Argentino se encuentran los códigos de fondo (Civil, Penal, Comercial) y que nacen del art. $41^{\circ}$ de la Constitución Nacional.

En el cuarto nivel se hallan la demás normativa, provincial o municipal. ${ }^{3}$

El Protocolo Adicional a la Convención Interamericana sobre Derechos Humanos en materia de Derechos Económicos, Sociales y Culturales, «Protocolo de San Salvador» (Ratificada por Ley 24658):

Art. 10.- Derecho a la salud

Toda persona tiene derecho a la salud, entendida como el disfrute del más alto nivel de bienestar físico, mental y social (...).

Art. 11.- Derecho a un medio ambiente sano

1. Toda persona tiene derecho a vivir en un medio ambiente sano y a contar con servicios públicos básicos.

Es por ello que el documento final de la Conferencia afirma:

8. Reafirmamos también la importancia de la libertad, la paz y la seguridad, el respeto de todos los derechos humanos, entre ellos el derecho al desarrollo y el derecho a un nivel de vida adecuado, ${ }^{4}$ incluido el derecho a la alimentación, el estado de derecho, la igualdad entre los géneros, el empoderamiento de las mujeres y el compromiso general de lograr sociedades justas y democráticas para el desarrollo.

121. Reafirmamos nuestros compromisos relativos al derecho humano al agua potable y el saneamiento, que ha de hacerse efectivo gradualmente en beneficio de nuestra población, respetando plenamente nuestra soberanía nacional. Resaltamos también nuestro

${ }^{3}$ Vega, Juan Carlos (2006), “Los Derechos Humanos: Idea política, metodología de análisis crítico, legalidad supranacional” en Derechos Humanos, Legalidad y Jurisdicción Supranacional, Mediterránea, Córdoba, págs. 70-71.

${ }^{4}$ Equivalente a la expresión “ambiente sano” establecido en el art. $41^{\circ}$ de la CN. 
compromiso con el Decenio Internacional para la Acción "El agua, fuente de vida”, 2005-2015.

Por si ello fuera poco el proyecto de unificación de Código Civil y Comercial, enviado por el P.E. Nacional para su tratamiento por el Congreso Nacional, en concordancia con dicho razonamiento establece:

ARTÍCULO $1^{\circ}$.- Fuentes y aplicación. Los casos que este Código rige deben ser resueltos según las leyes que resulten aplicables. La interpretación debe ser conforme con la Constitución Nacional y los tratados en los que la República sea parte (...).

ARTÍCULO $2^{\circ}$.- Interpretación. La ley debe ser interpretada teniendo en cuenta sus palabras, sus finalidades, las leyes análogas, las disposiciones que surgen de los tratados sobre derechos humanos.

De manera que no queda duda alguna que los Derechos Ambientales, son Derecho Humanos fundamentales y cualquier interpretación que se haga de tales derechos debe realizarse conforme al sistema Internacional de los Derechos Humanos.

Va de suyo que este documento final de la Conferencia deberá servir de guía obligatoria para la interpretación de la legislación en nuestro país, en relación a los derechos allí protegidos.

${ }^{5}$ La Asamblea General de la ONU reconoció el 28 de julio de 2010 el acceso al agua potable como un derecho humano básico y urgió a garantizar que los casi 900 millones de personas que carecen del líquido vital puedan ejercer ese derecho. En una resolución adoptada por 122 votos a favor, ninguno en contra y 41 abstenciones, la Asamblea estipuló también que el acceso a los servicios sanitarios básicos es un derecho en vista de que la contaminación del agua es una de las principales causas de mortalidad en los países más pobres. En dicha Asamblea la representante de Argentina, ministra Ana María Bianchi explicó el voto a favor de su país diciendo: "La Argentina entiende que es una de las responsabilidades principales de los Estados asegurar a sus habitantes el derecho al agua como una de las condiciones fundamentales para garantizar el derecho a la vida y para asegurar un nivel de vida adecuado". En la resolución aprobada por dicha Asamblea, que lleva como título: "El derecho humano al agua y el saneamiento", se reconoce la importancia de disponer de agua potable y saneamiento en condiciones equitativas como componente integral de la realización de todos los derechos humanos y en tal sentido se reafirmó la responsabilidad de los Estados de promover y proteger todos los derechos humanos, que son universales, indivisibles, interdependientes y están relacionados entre sí y que deben tratarse en forma global y de manera justa y equitativa y en pie de igualdad y recibir la misma atención. 
VIII. Uno de los puntos más controvertidos es la cuestión de la denominada “economía verde”, la Declaración esboza las principales características de tal economía

58. Afirmamos que las políticas de economía verde en el contexto del desarrollo sostenible y la erradicación de la pobreza deberán:

a) Ser compatibles con el derecho internacional;

b) Respetar la soberanía nacional de cada país sobre sus recursos naturales, teniendo en cuenta sus circunstancias, objetivos, responsabilidades, prioridades y margen de acción con respecto a las tres dimensiones del desarrollo sostenible;

c) Contar con el respaldo de un entorno propicio e instituciones que funcionen adecuadamente a todos los niveles, con la asignación de una función de liderazgo a los gobiernos y con la participación de todos los interesados pertinentes, incluida la sociedad civil;

d) Promover el crecimiento económico sostenido e inclusivo, fomentar la innovación, ofrecer oportunidades, beneficios $y$ empoderamiento para todos y respetar los derechos humanos;

e) Tener en cuenta las necesidades de los países en desarrollo, en particular los países en situaciones especiales;

f) Fortalecer la cooperación internacional, la cual incluye el suministro de recursos financieros y la transferencia de tecnología a los países en desarrollo y el fortalecimiento de su capacidad;

g) Lograr evitar la imposición de condiciones injustificadas a la asistencia oficial para el desarrollo y la financiación;

h) No constituir un medio de discriminación arbitraria o injustificable ni una restricción encubierta del comercio internacional, evitar la adopción de medidas unilaterales para afrontar los desafíos ambientales fuera del ámbito de jurisdicción del país importador, y asegurar que las medidas ambientales destinadas a solventar los problemas ambientales transnacionales o mundiales se basen en un consenso internacional, en la medida de lo posible;

i) Contribuir a colmar la brecha tecnológica entre los países desarrollados y en desarrollo y reducir la dependencia tecnológica de los países en desarrollo, adoptando todas las medidas oportunas; j) Mejorar el bienestar de los pueblos indígenas y sus comunidades, otras comunidades locales y tradicionales y las minorías étnicas, reconociendo y apoyando su identidad, cultura e intereses, y evitar poner 
en peligro su patrimonio cultural, sus prácticas y sus conocimientos tradicionales, preservando y respetando los enfoques no orientados al mercado que contribuyan a la erradicación de la pobreza;

k) Mejorar el bienestar de las mujeres, los niños, los jóvenes, las personas con discapacidad, los pequeños agricultores y los agricultores de subsistencia, los pescadores y quienes trabajan en empresas pequeñas y medianas, y mejorar los medios de vida y el empoderamiento de los pobres y los grupos vulnerables, en particular en los países en desarrollo;

l) Aprovechar al máximo el potencial de las mujeres y los hombres y asegurar que su contribución se realice en condiciones de igualdad; $m)$ Promover actividades productivas en los países en desarrollo que contribuyan a la erradicación de la pobreza;

n) Atender las preocupaciones que suscitan las desigualdades y promover la inclusión social, incluidos niveles mínimos de protección social;

o) Promover modalidades sostenibles de consumo y producción;

p) Perseverar en el empeño de superar la pobreza y la desigualdad mediante.

63. Reconocemos la importancia de evaluar todos los factores sociales, ambientales y económicos y alentamos su integración en el proceso de adopción de decisiones, cuando las circunstancias y condiciones nacionales lo permitan.

Reconocemos que será importante tener en cuenta las oportunidades y los desafíos, así como los costos y los beneficios, de las políticas de economía verde en el contexto del desarrollo sostenible y la erradicación de la pobreza, empleando los mejores datos y análisis científicos disponibles. Reconocemos que la aplicación de una combinación de medidas a nivel nacional y en consonancia con las obligaciones contraídas en virtud de acuerdos internacionales, incluidas medidas reguladoras, voluntarias y de otra índole, podría promover la economía verde en el contexto del desarrollo sostenible y la erradicación de la pobreza. Reafirmamos que las políticas sociales son esenciales para promover el desarrollo sostenible.

Ello necesariamente deberá reflejarse en instrumentos superadores del proceso de evaluación de impacto ambiental a fin de utilizar otras herramientas más completas a saber: 
Evaluación de Impacto Social y Ambiental (ESI): En ello se estudia los impactos sociales y ambientales como una unidad inseparable, ya que toda obra implica consecuencias sociales en la comunidad donde se planifica la misma y sus posibles impactos negativos.

Evaluación del Impacto Social (EIS): El énfasis de las primeras EIS fue en los aspectos biofísicos (en años más recientes, la práctica de la EIS ha interpretado al medioambiente de manera más holística). Como resultado, la EIS surgió como técnica para atender explícitamente las dimensiones sociales en la evaluación de proyectos. Se ha usado como enfoque independiente, o en una aplicación más integrada como la Evaluación de Impacto Ambiental y Social. Esto reviste particular importancia en la cooperación para el desarrollo, en donde se han articulado bien las interrelaciones entre pobreza/desarrollo y medioambiente.

Evaluación de Riesgo (Risk Assessment): Como decíamos vivimos en una sociedad de riesgo, de allí que es importante el estudio acabado de los posibles riesgos que por ejemplo traería la introducción en el ambiente o en la salud de la población elementos que fueron biogenéticamente manipulados.

Evaluación del Impacto Acumulativo (EIAc): La EIA de una propuesta de proyecto específica puede fallar en considerar sus potenciales impactos agregados, incrementales y sinérgicos con otros proyectos, en un programa de desarrollo que cubra un área completa. La EIAc es un subconjunto de la EAE, en desarrollo, que evolucionó para captar estas implicaciones más amplias en la evaluación de proyectos. Implicaciones capaces de cambiar las conclusiones de una evaluación de proyecto individual. Un proyecto al que se le atribuye pocos o insignificantes impactos — considerado aisladamente— puede resultar con impactos potenciales de gran relevancia considerado como parte de un conjunto de desarrollo más complejo. La EIAc representa entonces un desplazamiento hacia arriba en la jerarquía de toma de decisiones, aunque en el nivel de programa/plan, y no de políticas.

Evaluación de Sostenibilidad Ambiental: Es aquella que se requiera por ejemplo para la integración de bloques económicos de países, como paso previo a su realización (por ej. Mercosur- Unión Europea; Alca- Mercosur).

Este tipo de evaluación está prevista para evaluar la mayoría de los efectos tanto ambientales, sociales, políticos, de cumplimiento de las leyes laborales, etc. ${ }^{6}$

${ }^{6}$ A manera de ejemplo, Walsh, Juan Rodrigo; Ortiz, Eduardo \& Galperín, Carlos, Sostenibilidad en el Comercio: Evaluación de los Impactos Potenciales del ALCA, El Caso de Argentina, FIDA; FARN; North South Center y Tulane Institute for Environmental Law \& Policy, EEUU, septiembre de 2003. 


\section{La Evaluación Ambiental Estratégica:}

Las EAEs son procedimientos formales y sistemáticos que ayudan a identificar los impactos ambientales que pueden surgir de acciones más amplias tales como nuevas políticas, planes de desarrollo regional o nacional e iniciativas de programas importantes. Ellas ayudan a introducir las acciones y las consideraciones ambientales dentro del proceso de toma de decisiones por encima y más allá del nivel del proyecto. (World Bank: 1997).

Nuestra legislación la establece en la Ley 26639 de presupuestos mínimos de Protección de Glaciares y del Ambiente Periglacial.

Ley 26639:

ARTÍCULO $7^{\circ}$ - Evaluación de impacto ambiental. Todas las actividades proyectadas en los glaciares y en el ambiente periglacial, que no se encuentran prohibidas, estarán sujetas a un procedimiento de evaluación de impacto ambiental y evaluación ambiental estratégica, según corresponda conforme a su escala de intervención, en el que deberá garantizarse una instancia de participación ciudadana de acuerdo a lo establecido en los artículos 19, 20 y 21 de la Ley $N^{\circ} 25.675$ —Ley General del Ambiente-, en forma previa a su autorización y ejecución, conforme a la normativa vigente.

El alcance de la EAE está definido en el Dec. 207/2011 reglamentario de la Ley 26639 que, establece:

Art. $7^{\circ}$ : Se entiende por Evaluación Ambiental Estratégica aquel proceso sistemático de estudio de impacto ambientales de las políticas, planes y programas y de sus alternativas, incluyendo la preparación de un informe escrito y las conclusiones de su evaluación y su uso en los procesos de decisiones públicas.

$I X$. En relación al plano internacional el documento resalta la función del PUNUMA (Programa de las Naciones Unidas para el Medio Ambiente)

87. Reafirmamos la necesidad de fortalecer la gobernanza ambiental internacional en el marco institucional para el desarrollo sostenible, a fin de promover la integración equilibrada de las dimensiones económica, social y ambiental del desarrollo sostenible, así como la coordinación en el sistema de las Naciones Unidas. 
88. Nos comprometemos a fortalecer la función del Programa de las Naciones Unidas para el Medio Ambiente (PNUMA) como principal autoridad ambiental mundial que establece las actividades mundiales en pro del medio ambiente, promueve la aplicación coherente de los aspectos ambientales del desarrollo sostenible en el sistema de las Naciones Unidas y actúa como defensor autorizado del medio ambiente a nivel mundial. Reafirmamos la resolución 2997 (XXVII), de 15 de diciembre de 1972, en la que se estableció el PNUMA, y otras resoluciones pertinentes que refuerzan su mandato, así como la Declaración de Nairobi sobre el papel y el mandato del PNUMA, de 1997, y la Declaración Ministerial de Malmö, de 2000 .

Por otro lado se recalca la importancia de los convenios internacionales sobre sustancias peligrosas.

89. Reconocemos las importantes contribuciones de los acuerdos multilaterales sobre el medio ambiente al desarrollo sostenible. Reconocemos la labor iniciada para mejorar las sinergias entre los tres Convenios sobre los productos químicos y los desechos (el Convenio de Basilea sobre el control de los movimientos transfronterizos de los desechos peligrosos y su eliminación, el Convenio de Rotterdam sobre el Procedimiento de Consentimiento Fundamentado Previo Aplicable a Ciertos Plaguicidas y Productos Químicos Peligrosos Objeto de Comercio Internacional y el Convenio de Estocolmo sobre contaminantes orgánicos persistentes). Alentamos a las partes en los acuerdos multilaterales sobre el medio ambiente a que consideren la posibilidad de adoptar otras medidas relacionadas con estos y otros grupos de cuestiones, cuando proceda, para promover la coherencia de las políticas en todos los niveles, mejorar la eficiencia, reducir las superposiciones y duplicaciones innecesarias y mejorar la coordinación y la cooperación entre los acuerdos, en particular el Convenio y las dos Convenciones de Río, así como con el sistema de las Naciones Unidas sobre el terreno.

$X$. En relación al Marco para la acción y el seguimiento y a las esferas temáticas y cuestiones intersectoriales, el documento analiza numerosos temas, a saber: 
i) Erradicación de la pobreza

ii) Seguridad alimentaria y nutrición y agricultura sostenible

iii) Agua y saneamiento

iv) Energía

v) Turismo sostenible

vi) Transporte sostenible

vii) Ciudades y asentamientos humanos sostenibles

viii) Salud y población

En tal sentido solamente analizaré dos puntos que considero esenciales, el primero de ellos referidos a la salud y el segundo al problema poblacional:

138. Reconocemos que la salud es una condición previa, un resultado y un indicador de las tres dimensiones del desarrollo sostenible. Sabemos que solo se podrán lograr los objetivos del desarrollo sostenible cuando no haya una alta prevalencia de enfermedades debilitantes transmisibles y no transmisibles y las poblaciones puedan llegar a un estado de bienestar físico, mental y social. Estamos convencidos de que las medidas sobre los determinantes sociales $y$ ambientales de la salud, tanto para los pobres y vulnerables como para toda la población, son importantes para crear sociedades inclusivas, equitativas, económicamente productivas y sanas. Pedimos que se logre plenamente el derecho de todos a disfrutar del más alto nivel posible de salud física y mental.

145. Pedimos que se apliquen plena y efectivamente la Plataforma de Acción de Beijing, el Programa de Acción de la Conferencia Internacional sobre la Población y el Desarrollo y los resultados de sus conferencias de examen, incluidos los compromisos relativos a la salud sexual y reproductiva y la promoción y protección de todos los derechos humanos en este contexto. Ponemos de relieve la necesidad de proporcionar acceso universal a la salud reproductiva, incluida la planificación familiar y la salud sexual, y de integrar la salud reproductiva en las estrategias y los programas nacionales.

$I X$. Promoción del empleo pleno y productivo, el trabajo decente para to- 
dos y la protección social

x) Océanos y mares

xi) Pequeños Estados insulares en desarrollo

xii) Países menos adelantados

xiii) Países en desarrollo sin litoral

xiv) África

$\mathrm{xv}$ ) Iniciativas regionales

xvi) Reducción del riesgo de desastres

xvii) Cambio climático

xviii) Bosques

xix) Biodiversidad

xx) Desertificación, degradación de la tierra y sequía

xxi) Montañas

xxii) Productos químicos y desechos

xxiii) Consumo y producción sostenibles

xxiv) Minería

xxv) Educación

xxvi) La igualdad entre los géneros y el empoderamiento de las mujeres

XI. En relación a las fuentes de financiamiento del Desarrollo Sostenible el documento resalta:

253. Exhortamos a todos los países a que prioricen el desarrollo sostenible en la asignación de recursos según las prioridades y necesidades nacionales, y reconocemos la importancia fundamental de aumentar el apoyo financiero de todas las fuentes para el desarrollo sostenible de todos los países, en particular los países en desarrollo. Reconocemos la importancia de los mecanismos financieros internacionales, regionales y nacionales, incluidos los que sean accesibles a las autoridades subnacionales y locales, para implementar programas de desarrollo sostenible y pedimos su fortalecimiento e implementación. Las nuevas asociaciones y las fuentes innovadoras de financiación pueden servir de complemento a las fuentes de financiación para el desarrollo sostenible. Alentamos que se sigan examinando y utilizando, junto con los medios tradicionales de ejecución. 
258. Reconocemos que es crucial que se respeten todos los compromisos relacionados con la AOD, como los contraídos por numerosos países desarrollados de alcanzar, para 2015, el objetivo de dedicar el 0,7\% del producto nacional bruto (PNB) a la AOD de los países en desarrollo, así como el objetivo de dedicar entre el 0,15\% y el 0,20\% del PNB a los países menos adelantados. A fin de cumplir los calendarios convenidos, los países donantes deberían tomar todas las medidas necesarias y apropiadas para elevar la tasa de desembolsos de la ayuda con objeto de que cumplan sus compromisos actuales. Instamos a los países desarrollados que aún no lo hayan hecho a que hagan más esfuerzos concretos para alcanzar el objetivo de dedicar el 0,7\% del PNB a la AOD de los países en desarrollo, incluida la meta específica de dedicar entre el 0,15\% y el 0,20\% del PNB a los países menos adelantados, de conformidad con sus compromisos. A fin de aprovechar los progresos logrados en la labor de garantizar que la AOD se utilice eficazmente, destacamos la importancia de la gobernanza democrática, una mayor transparencia y rendición de cuentas y la gestión orientada hacia los resultados. Alentamos encarecidamente a todos los donantes a que elaboren, cuanto antes, calendarios indicativos móviles que ilustren cómo piensan alcanzar sus metas, de conformidad con sus respectivos procesos de consignación presupuestaria. Destacamos la importancia de movilizar un mayor apoyo nacional en los países desarrollados para el cumplimiento de sus compromisos, por ejemplo, mediante la sensibilización pública, facilitando datos respecto a los efectos de la ayuda sobre el desarrollo y demostrando resultados tangibles.

XII. El documento también hace referencia a la importancia de la transferencia de la tecnología y la capacitación para el Desarrollo sostenible; a las reglas del comercio internacional y la misión en tal sentido de la OMC (Organización Mundial del Comercio).

Finalizando con una exhortación al registro de compromisos asumidos por todos los países participantes en la Conferencia.

XIII. Colofón: Participamos de la opinión que el Derecho Ambiental es 
invasivo de las demás ramas del Derecho.

Este documento internacional profundiza tal principio y más allá de las críticas que podrá recibir es, una base sólida para que los Derechos Ambientales, Derechos Humanos fundamentales de todos los habitantes de nuestro planeta puedan ser ejercidos efectivamente.

Todo ello sin dejar de mencionar que todos los seres humanos tenemos prioritariamente- para con el ambiente deberes y obligaciones que tenemos que cumplir si queremos sobrevivir, nosotros y las generaciones futuras. 